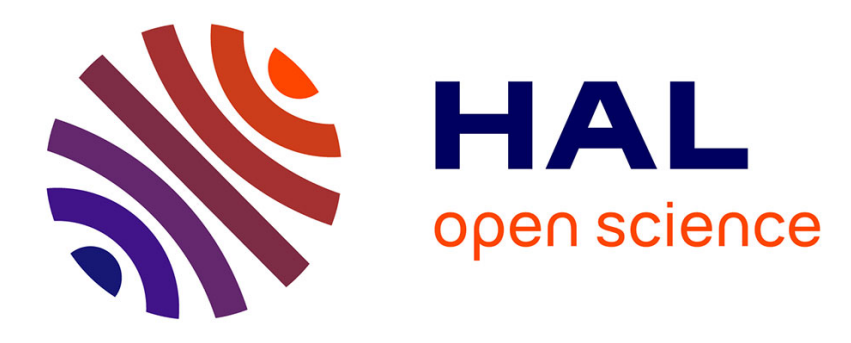

\title{
L'héritage pré et post-romain dans les réseaux routiers anciens
}

\author{
Sandrine Robert
}

\section{To cite this version:}

Sandrine Robert. L'héritage pré et post-romain dans les réseaux routiers anciens. Les Nouvelles de l'archéologie, 2009, 115, pp.23-30. halshs-00442792

\section{HAL Id: halshs-00442792 \\ https://shs.hal.science/halshs-00442792}

Submitted on 22 Dec 2009

HAL is a multi-disciplinary open access archive for the deposit and dissemination of scientific research documents, whether they are published or not. The documents may come from teaching and research institutions in France or abroad, or from public or private research centers.
L'archive ouverte pluridisciplinaire HAL, est destinée au dépôt et à la diffusion de documents scientifiques de niveau recherche, publiés ou non, émanant des établissements d'enseignement et de recherche français ou étrangers, des laboratoires publics ou privés. 


\title{
L'héritage pré et post-romain dans les réseaux routiers anciens
}

\author{
S. Robert,
}

Maître de conférence associé Université de Paris I, UMR 7041 Arscan Nanterre et Conseil général du Val-d’Oise

\begin{abstract}
Résumé
L'apport des périodes pré et post-romaines dans la construction et la transmission des réseaux routiers peut être réévalué aujourd'hui grâce à l'archéologie préventive et l'archéogéograhie qui permet de hiérarchiser l'information très riche collectée sur le terrain. La route protohistorique transparaît à travers des découvertes archéologiques de plus en plus nombreuses et surtout au rôle qu'elle joue dans la structuration du paysage. Son héritage dans le paysage antique est important. La route médiévale n’apparaît plus uniquement en rupture avec le réseau antique. Les cas de transmission sont nombreux mais il existe aussi des rééquilibrages en fonction des nouveaux pôles émergents.
\end{abstract}

Mots-clés : routes protohistoriques et médiévales, parcellaire, paysage, transmission

Abstract

Today, we can use preventive archaeology and archaeogeography to assess the decisive contribution of periods before and after antiquity in the construction and transmission of road networks. Datas collected on archaeological excavations are numerous and can be classified depending on their scale by archaeogeography. Protohistoric roads are well known thanks to archaeological discoveries and thanks to their role in landscape structuration shown by archaeogeography studies. Its heritage in antic landscape is decisive. There's no hiatus between antic and medieval road network. There are many transmission but the emergence of new centres brings new directions.

Key-words : protohistoric and medieval roads, plots, landscape, transmission

\section{Introduction}

Le «monopole » de la recherche sur les voies antiques a focalisé l'attention sur les tracés et modelés monumentaux qui ne rendent pas compte de la complexité des réseaux routiers dans le temps (cf. S. Robert infra). Aujourd'hui, pour passer d'une vision de la route comme objet à celle d'un réseau associant structure physique, trafic et phénomènes de transmissions à différentes échelles, il est nécessaire de réévaluer le rôle des périodes pré et post-romaines. Là où l'archéologie ou les sources écrites font défaut ou ne permettent qu'une vision très fragmentaire, l'archéogographie permet d'étudier le réseau à petite échelle et de hiérarchiser les observations de terrain.

\section{I - L’héritage pré-romain}

\section{I- 1 : Histoire de la recherche}

Chez les protohistoriens, la réseau de circulation a été abordé surtout à partir de la distribution des artefacts. La mise en évidence du réseau physique, prépondérante chez les antiquisants, s'est effacée derrière la fonction : la circulation des biens, des hommes et des objets. Pour les échanges, attestés depuis le néolithique à travers la circulation des matières premières ou des types stylistiques, ce sont surtout les voies navigables qui sont évoquées (Fustier 1960, p. 135, Vendryès 1957). Seules quelques découvertes ponctuelles attestent de l'existence de chemins construits en dur dès l'Âge du Bronze et pour l'Âge du fer, les chercheurs ont fait le même constat de la difficulté à saisir le réseau routier : il est supposé existant plus que véritablement 
connu par des évidences archéologiques (Chevallier (dir.) 1983, Malrain et al. 2002 : 25). Son importance est déduite notamment de la vitesse de déplacement des belligérants et d'allusions à des ponts gaulois dans les Commentaires de César sur la Guerre des Gaules (Chevallier 1972). Par ailleurs, la richesse de l'artisanat de la charronnerie et de la bourrellerie et du vocabulaire associé laisse supposer une activité importante en liaison avec la route (Guillaumet 1996: 61 et 71). Une localisation par rapport aux tumuli et aux oppida a été tentée dans le Limousin et le Périgord (Desbordes et Barrière 1983), chez les Rèmes (Legros 1983), et le long du Rhône (Chapotat 1981), etc.

\section{I- 2 : La réalité matérielle des chemins protohistoriques}

Mais l'aspect matériel du réseau pré-romain n’a été perçu que ponctuellement à partir des découvertes de terrain et des prospections aériennes. À partir des années 1970, des chemins d'origine gauloise supposée ont été suivis sur plusieurs centaines de mètres en Bretagne, dans les Ardennes et en Picardie. À Liercourt, Vers-sur-Selle, Bray-lès-Mareuil, Dreuil-Hamel, et Cagny, R. Agache a pu mettre en évidence des chemins creux, bordés de fossés, associés à des fermes gauloises (Agache 1978 : 198, 199, 142, 147, 204). Il décrit ces voies gauloises ou de tradition gauloise comme "assez sinueuses avec un ou deux fossés latéraux ». Parfois, elles sont jalonnées de "curieuses grandes tâches sombres qui correspondent soit à des élargissements pour faciliter des croisements, soit plus vraisemblablement à d'anciennes carrières » (Agache 1978 : 204). Certains tracés ont été confirmés par la fouille. À AcyRomance, un chemin franchissant l'Aisne à Nanteuil-sur-Aisne était large de 2,10 à 4m et présentait des ornières d'un écartement de 1,50m. Il a livré un mobilier de La Tène D1 mais il peut être d'une origine peut-être plus ancienne : Bronze final ou Tène ancienne (Lambot 1996 : 25). Ces dernières années, les opérations archéologiques couvrant plusieurs hectares ont été l'occasion de multiplier les observations sur ces portions de chemins pouvant être rattachés à la protohistoire et les archéologues ont souligné leur rôle dans la structuration de l'habitat et du parcellaire.

À Marguerittes, près de Nîmes, un chemin associé à un habitat du Ve siècle av. J. C. a été fouillé en 1995. Large de 1,80 à 4m et bordé de fossés, il comportait une bande de roulement empierrée sur laquelle des ornières ont été observées (Vidal et al. 1996 : 62). Au Viol du Plan, un carrefour antique dont un des axes serait hérité d'un chemin protohistorique a été mis en évidence. Les archéologues notent que ce chemin a été un morphogène important dans la structure parcellaire jusqu’à récemment (Vidal et al. 1996 : 63-64). Sur 113 hectares explorés près de Montpellier, A. Chartrain relève une importante densité de chemins souvent en relation avec des nécropoles. Il signale qu'une voie rurale sur quatre date de l'Âge du Fer et que « cette période est donc contre toute attente beaucoup moins discrète dans ce domaine que dans celui des sites ou des habitats ». Ces chemins perdurent souvent aux périodes postérieures. Sur la ZAC Mermoz, dès la période républicaine, deux fossés parallèles et distants de 6,50 m matérialisent un chemin qui passe entre deux nécropoles. Au Haut Empire, un chemin en reproduit le tracé (dont la largeur est portée à $11 \mathrm{~m}$ ) tout en s’intégrant à l'orientation de la cadastration Nîmes A (Chartrain 2007).

Si les découvertes publiées sont peu nombreuses, un rapide examen des rapports d'opérations archéologiques sur les grands travaux montre que des vestiges de chemins protohistoriques sont rencontrés sur le terrain. Dans le cadre du PCR Dynarif, L. Gonzalo-Valencia a relevé les mentions de voies, chemins ou fossés bordiers sur les documents finaux de synthèse de fouilles et diagnostics réalisés par l'INRAP en Ile-de-France depuis 2005. Sur les 70 mentions, la protohistoire représente $7 \%$ des $\operatorname{cas}^{1}$ (cf. Fig. X). Il s'agit le plus souvent de

1 L. Gonzalo-Valencia : «La base de données du centre archéologique de Pantin », in : S. Robert et N. Verdier 
chemins en structure légère et qui peuvent passer inaperçus lors des sondages et des fouilles. Les datations sont souvent déduites de leur relation avec des structures mieux datées (fossés d'habitat ou de parcellaire).

A. Grenier décrivait dès 1934 des voies à « structure légère » simples chemins de terre, ou revêtues de gravier ou de scories, bordées ou non de fossés (Grenier 1985: 345-354 et 389391). Il précisait qu' "elles paraissent bien souvent n’être autres que d'anciens chemins gaulois, entretenus à la mode indigène » (Grenier 1985: 391). Il signalait que ce type de voies était mentionné dans le Code de Justinien. Un texte d'Ulpien distinguait trois sortes de routes : d'abord la via terrena, simple chemin de terre ou " piste devenue route par l'usage » pour A. Grenier, ensuite la via glarea strata, voie recouverte de gravier et enfin la via lapide strata : voie pavée ou dallée (Digeste, 43, 11 transcrit par Grenier 1985: 332). Les historiens insistèrent surtout sur la troisième catégorie.

Pour la Protohistoire, le réseau routier n’est donc pas totalement absent mais les découvertes doivent être repérées et surtout hiérarchisées. En effet, elles restent pour l'instant limitées à la fenêtre de fouille ou du cliché aérien. Il est dès lors nécessaire de changer d'échelle et de méthode d’observation pour associer ou non ces observations à des tracés supra-locaux voire régionaux.

Figure 1 : En 1934, A. Grenier attire l'attention sur les voies en structures légères qu'il pense héritées de la période gauloise (Grenier 1985 : 347).

\section{I - 3 : Voies protohistoriques et structuration du paysage}

Les analyses archéogéographiques croisées avec les données de terrain permettent de changer d'échelle d'observation pour mettre en évidence des axes cohérents sur plusieurs kilomètres, et observer leur relation avec d'autres éléments du paysage.

Dans le Tricastin, C. Jung a révélé grâce à la télédétection un chemin qui paraît relier l'oppida du Moulon sur la commune de Roussas (Drôme) et celui de Saint-Etienne-de-Dion sur la commune de Saint-Marcel-d'Ardèche (Ardèche). Son franchissement théorique sur le Rhône correspond à un haut-fond et de nombreux établissements gallo-romains jalonnaient cet itinéraire (Jung 1999).

Dans le Val-d'Oise, certaines voies mises en évidence par l'étude archéogéographique ont pu être mises en relation avec des établissements protohistoriques. L'oppidum de Nucourt, dont le rempart a été implanté à partir de la fin du Hallstatt final, début de la Tène ancienne, est situé au carrefour du Chemin des Gendarmes dans l'itinéraire Chartres-Beauvais et du Chemin aux Prêtres dans l'itinéraire Paris-Rouen. Ce dernier est aussi jalonné par deux sites protohistoriques importants : un habitat groupé gaulois associé à une nécropole à Epiais-Rhus et l'oppidum dit Camp de César à Frépillon (Robert 2006b). A Fontenay-en-Parisis, la Voirie Vendrelle entrant dans l'itinéraire Paris-Senlis a été fouillée sur le site de La Lampe. La voie matérialisée par " une série d'ornières encadrées par deux fossés, distants de $6 \mathrm{~m}$ d'axe à axe" a livré de la céramique du Ier s. ap. J. C. L’établissement qui la borde s’est caractérisé par la présence de banquets de la Tène Moyenne au Bas-Empire, et peut correspondre à un lieu de culte (Daveau et Yvinec 2002 : 131).

Ailleurs, c'est la relation entre des voies et des trames parcellaires datées qui suggère l'existence, avant la conquête, de voies de grand parcours imprimant une orientation dominante à de vastes trames parcellaires non orthonormées.

Dans la plaine des Tille et de l'Ouche en Côte d'Or, G. Chouquer signale la Voire

(coord.) - Projet Collectif de Recherche (PCR). Dynamique et résilience des réseaux routiers et parcellaires en région Ile-de-France (DYNARIF). CNRS, UMR 7041 Arscan et UMR UMR 8504, Géographie-cités. Service régional de l'archéologie d’Ile-de-France, Saint-Denis, décembre 2007 : 86-89 
Traversaine reliant Besançon et Chalon, bien attestée pour la période antique, mais dont « il y a tout lieu de penser qu'il s'agisse d'une voie gauloise pérennisée » (Chouquer et al. 1996 : 39). Elle structure un système parcellaire aujourd'hui en grande partie fossile. À Genlis, « Terre du Varin », la trame des voies et du parcellaire a été mise en relation avec une ferme gauloise en activité entre 120 et 50 av. J.-C. Si la période d'utilisation semble bien attestée pour la fin de l’Âge du fer et l'époque romaine, les auteurs pensent que la réification du système prend ses racines entre le Hallstatt et la fin du second age du fer. (Chouquer et al. 1996). G. Chouquer propose de rapprocher ce réseau des cohesive system mis en évidence dans la recherche anglo-saxone (Chouquer 2006).

À Sénart, un vaste réseau non orthonormé d'orientation est-ouest, nord-sud organisait l'ensemble du plateau. La comparaison avec les données de terrain a montré que cette orientation serait apparue dès la Tène Finale (Robert 1996). En Limagne, le même type de trame s'appuie sur des alignements remarquables dont l'un est formé par la voie antique Autum-Clermont-Saint-Paulien. Cette orientation est également présente dans un ensemble parcellaire qui se met en place à partir de La Tène C2 sur le site du « Patural » à ClermontFerrand (Mennessier-Jouannet et Buchsenschutz 1996).

Si ces grandes trames se construisent dans la longue durée, l'âge du fer paraît déterminant dans leur phase d'émergence et de structuration. On passerait d'une forme discontinue en îlots ou corridors à une occupation continue, avec des pôles connectés entre eux (Chouquer 2006). La densification de l'occupation du sol, la mise en valeur de terrains agricoles hydromorphes nécessitant un drainage et également la connexion à des axes de grand parcours pourraient avoir joué un rôle dans cette réification.

Les effets structurants des axes de circulation apparaissent aussi à l'étude des relations entre des sites protohistoriques. A. Chevalier a modélisé les plus courts chemins associés à des groupements de tumuli dans la plaine de Caen. Il a montré que la dispersion des tombes n'était pas aléatoire et qu'elle pouvait être mise en relation avec des axes de circulation dont certains sont connus des archéologues (cf. Chevalier infra).

Archéogéographie et analyse spatiale montrent donc l'importance du réseau viaire dans la structuration du paysage dès la période gauloise.

L'idée n'est pas nouvelle. Dès 1934, A. Grenier posait l'hypothèse que la plupart des voies antiques étaient antérieures à l'époque romaine. Il proposait de comprendre le réseau d'Agrippa - décrit par Strabon à la fin du règne d'Auguste - , non comme la création de nouveaux chemins mais comme la description de chemins pré-existants qui auraient été englobés dans des itinéraires administratifs et équipés pour être adaptés à cette nouvelle fonction. À coté des chemins officiellement choisis, subsisterait « l'ancien réseau gaulois, toujours en usage et, comme précédemment, utilisé par les indigènes » (Grenier 1985: 24 et 43-44). Cette idée avait été développée aussi par Camille Jullian qui avait remarqué que certaines chaussées Brunehaut aboutissaient à des oppida abandonnées à l'époque impériale plutôt qu'aux nouvelles fondations : la grande voie de Bavai à Paris visant Vermand plutôt que Saint-Quentin, par exemple (Jullian 1920 : 106).

G. Chouquer, devant l'importance jouée par la période laténienne dans la structuration des paysages, pense qu' " un fait paysager majeur est sans doute la création d'un réseau de grandes voies commerçantes, dès l'Âge du Fer, au point qu'il est envisageable que bon nombre de nos voies dites « romaines » soient, en fait, des voies gauloises. » (Chouquer et al. 1996 : 202).

Reste aujourd'hui à mieux percevoir la matérialité du réseau gaulois à partir d'inventaires plus systématiques des découvertes et de leur croisement avec les données de l'archéogéographie et de l'analyse spatiale pour changer d'échelle et les replacer dans de véritables réseaux de 
circulation (cf. S. Robert infra).

L’apport de la Protohistoire rétabli, il est plus aisé de concevoir la diversité du réseau antique, qui n'est pas constitué uniquement des grandes routes monumentales rectilignes mais également d'axes non arpentés (Robert 2006a). Pour appréhender le réseau routier dans la longue durée, reste à évaluer alors la part du Moyen-Âge dans sa transmission et dans sa transformation.

\section{II - La transmission post-romaine}

\section{II - 1 : Histoire de la recherche}

Les études archéogéographiques croisées avec les données de terrain ont montré qu’il était possible de percevoir les réseaux protohistoriques et antiques à travers les sources modernes et contemporaines. Le Moyen-Âge apparaît donc comme une période de transmission. Or, de l'aveu des historiens eux-mêmes, il fait figure de parent pauvre dans la recherche sur les routes (Derville 1978, Rouche 1982, Livet 2003, Bréchon 2000, Laffont et Bréchon 2008). De trop rares études ont associé systématiquement l'étude des textes et la reconstitution topographique des tracés routiers (Bautier 1987, Bréchon 2000). Comme pour la protohistoire, la part importante qui est accordée au réseau fluvial pour le transport à grande distance a pu contribuer à minimiser la recherche sur la route. Mais des questions idéologiques sous-tendent aussi cette absence.

Jusque dans les années 1970, le réseau routier médiéval est souvent pensé en rupture avec le réseau antique. On lit encore par exemple dans le Dictionnaire de la France médiévale :

"Le réseau routier du Moyen-Âge ne correspond que rarement au réseau gallo-romain, dont l'entretien a cessé à l'époque barbare et dont le tracé, souvent stratégique, est inadapté à l'usage des voyageurs du Moyen-Âge, et notamment des marchands. La relation immédiate de ville à ville l'emporte à partir du Xe siècle sur la relation lointaine qui régissait le tracé romain.» (Favier 1993 : 838). Pour le haut Moyen-Age, l'image d'une économie domaniale repliée sur elle-même a longtemps limité la recherche sur les échanges à grande distance (Bruand 2002). Le maintien du réseau routier régional ne pouvait que difficilement être pensé en dehors du maintien d'un pouvoir politique fort et centralisé. Il s'inscrivait donc en faux dans le contexte de désagrégement du pouvoir étatique et du morcellement des politiques territoriales du premier Moyen-Âge, observés par les historiens. L’image de l'émergence de réseaux routiers rayonnant en étoile autour de nouveaux centres locaux et servant de base à une réorganisation radio-concentrique du parcellaire s'accordait mieux avec les théories de l'incastellamento ou de l'encellulement (Watteaux 2004).

Pourtant, dès 1982, dans les actes du colloque de Flaran 2 consacré à « L'Homme et la Route en Europe occidentale au Moyen-Âge et aux Temps modernes », M. Rouche rétablissait la part importante de l'héritage antique dans la voirie médiévale et signalait que le haut MoyenÂge constituait un véritable " chaînon manquant » dans la recherche historiographique sur les routes (Rouche 1982 : 13). Il montrait que les voies romaines et certains relais et auberges étaient toujours utilisés à cette période. Avec le déplacement des centres de décision politiques vers le nord et l'est, le trafic s'était ré-orienté mais utilisait toujours le réseau principal. Certaines routes romaines secondaires seraient devenues principales, tandis que d'autres auraient été déclassées (Rouche 1982 : 18). Le IXe siècle est ainsi considéré comme une période de "remise en ordre de l'ancien réseau routier romain " au service notamment des missi dominici (Rouche 1982 : 20). Le réseau romain se perpétuerait " par plaques dans les grandes plaines ou en étoiles autour des villes » tout en coexistant avec des routes nouvellement construites (Rouche 1982 : 30). Cet auteur propose de voir une phase de rupture 
à partir du Xe-XIe siècle, consécutive aux invasions scandinaves et surtout au développement des principautés territoriales au détriment d'un État fort garant de l'entretien des routes (Rouche 1982 : 32).

La difficulté à cerner le réseau routier médiéval réside dans le fait que les mentions textuelles sont rares et très dispersées (Bréchon 2000). Une classification juridique des chemins à partir de leur largeur a été couchée sur le papier en 1283 par Ph. Beaumanoir dans ses Coutumes du Beauvaisis mais G. Hubrecht y voit surtout un texte très empreint des écrits romains plus qu'une image de la réalité matérielle routière de la fin du XIIIe siècle ${ }^{2}$. Pourtant, la circulation à grande distance est relativement présente dans les textes à travers le déplacement des biens, des idées et des personnes : routes de pèlerinage, chemins sauniers, chemins de chasse-marée, chemins de troupeaux etc., voyages de prélats, de commerçants, circulation des nouvelles etc. et il fait l'objet de nombreuses recherches historiques (cf. Flaran 1982 et Voyages et Voyageurs au Moyen-Âge 1996 par exemple). Dans les années 1980, J. P. Devroey et S. Lebecq ont montré par les textes que la pratique de grands déplacements continentaux par charrois était présente au haut Moyen-Age, laissant supposer que "la voirie héritée a au moins continué d'être praticable » (Lebecq 1989: 420, Devroey 1984). Cette recherche a donné lieu à une cartographie à petite échelle, d'axes théoriques reliant des points localisés par les textes (cf. par exemple, la cartographie des routes théoriques entre les ateliers d'origine du trésor de Glisy à l'échelle du Bassin parisien par S. Lebecq dans son article « La Neustrie et la mer » : Lebecq 1989).

En 1982, T. Dunin-Wasowicz montrait l'intérêt de croiser l'étude des sources écrites avec l'étude régressive, les données archéologiques et une analyse de la relation route-habitat (Dunin-Wasowicz 1982). Dans sa thèse, F. Bréchon a proposé une mise en relation systématique des mentions textuelles avec les données cartographiques et archéologiques. En cartographiant les mentions textuelles directes de chemins médiévaux, croisées avec les données du cadastre napoléonien, il a proposé une reconstitution de près de 2000 kilomètres de routes majeures ou importantes en Vivarais et sur ses marges, à la fin du Moyen-Âge. Sans surprise, son étude montre une forme anastomosée du réseau qui se présente comme un faisceau de tracés parallèles joignant quelques itinéraires principaux. Il montre aussi qu'au haut Moyen-Âge, on utilise encore le réseau routier antique mais qu'apparaissent de nouveaux chemins pour desservir des régions émergentes aux siècles carolingiens. À partir du xiI ${ }^{\mathrm{e}}$ siècle, un tri s'opère, privilégiant les routes orientées suivant les réseaux commerciaux consolidés ou qui se développent. Les autres tronçons subsistent, mais sont réduits au rang d'axes locaux (Bréchon 2000). La relation avec les châteaux est dynamique : le château cherche la proximité de la route ou la " capture » parfois lorsqu'il présente un certain intérêt commercial. Plus qu'une implantation stratégique systématique qui lui paraît anachronique pour cette époque, P. Y. Laffont propose d'y voir l'intérêt pour les pouvoirs locaux d'exploiter des droits de péages et des débouchés commerciaux (Laffont et Bréchon 2008).

\section{II - 2 : La réalité matérielle des voies médiévales}

À l’instar des textes, l'archéologie a été jusqu'à aujourd'hui peu bavarde concernant la réalité matérielle des réseaux routiers médiévaux. Les observations de terrain, moins spectaculaires que pour l'Antiquité ont souvent montré une route fragile, sensible aux intempéries. R. Agache à partir d'exemples perçus en prospection aérienne, indique que les grandes routes médiévales se caractérisent par de « larges pattes d'oies dans toutes les montées » dues au fait qu'elles n'étaient pas empierrées et que les charrois passaient sur les côtés pour ne pas s'enliser (Agache 1978 : 197, 203-204). Dans le Vivarais, des chemins à ornières espacées de

2 Ph. De Beaumanoir - Coutumes de Beauvaisis, ed. Am. Salmon, Paris, 1899, réédité à Paris, chez les éditions A. et J. Picard, 1970 et 1974, ajouté d'un tome III : G. Hubrecht - Commentaire historique et juridique. 
1,20 étaient traditionnellement associés à la période médiévale mais $\mathrm{F}$. Bréchon précise que rien ne permet de les dater précisément et de les associer à de la circulation de grands parcours, les charrois étant utilisés aussi pour les déplacements locaux. Dans tous les cas, il est difficile de déduire l'importance du chemin de sa matérialité. Les routes médiévales du Vivarais, empruntées surtout par les caravanes muletières, sont des structures peu aménagées n'ayant laissé des traces monumentales que dans certains passages difficiles: murs de soutènement, ponts... (Bréchon 2000 : 50-51). La route médiévale lorsqu'elle ne s'appuie pas sur un axe monumental antique est difficilement saisissable sur le terrain. Comme en Protohistoire, faute de théorisation, il est difficile de la reconnaître d'après sa seule structure matérielle. Il faut alors croiser les observations de terrain, avec les sources écrites et la reconstitution du réseau à travers l'étude archéogéographique.

Pourtant, les découvertes sont nombreuses sur les chantiers d'archéologie préventive depuis quelques années : 16 sur 70 soit $23 \%$ des observations mentionnées sur les rapports d'opération de l'INRAP d'Ile-de-France depuis 2005 concerne le Moyen-Âge ${ }^{3}$ (cf. Figure 2).

Figure 2 : L’indexation des DFS INRAP entre 2005 et 2007 en Ile-de-France rééquilibre la part du Moyen-Age et celle de la Protohistoire dans la découverte de chemins en comparaison avec la base Mérimée (cf. Robert infra).

Elles montrent des structurations diverses, du simple chemin de terre à des structures empierrées. Dans certains cas, on a pu croiser ces observations de terrain avec l'analyse archéogéographique et ainsi les associer à des cheminements de grand parcours ou non.

À Montboucher, les Hayes, les archéologues ont observé le chemin Gontardin ou Costardin traversant le bassin de la Valdaine sur une vingtaine de kilomètre entre Montelinar et Manas. Ce chemin est cité dans les textes médiévaux et succède à une chaussée antique large de $2 \mathrm{~m}$ construite de galets et blocs de grès et encadrés de fossés bordiers. La chaussée médiévale, postérieure au XIIe siècle, est composée d'une couche de petits galets et graviers sur une dizaine de centimères d'épaisseur et 1,5m de large. Un seul fossé la borde (Jung 1999 : 105).

À Villiers-le-Sec, la voirie de Montmorency, entrant dans l'itinéraire Paris-Amiens et l'Avenue de Beaumont (avec sa variante, le chemin de Villaines) entrant dans l'itinéraire Beauvais-Meaux ont été fouillés par F. Gentili ${ }^{4}$. Ces chemins ont été relevés sur plusieurs kilomètres lors de l'analyse archéogéographique. Ils prennent peu en compte la topographie au profit d'une relation relativement directe entre des pôles régionaux ou intermédiaires (ex. l'Avenue de Beaumont à Beaumont-sur-Oise). Cependant, ils présentent un tracé sinueux qui ne peut être comparé au tracé rectiligne des axes arpentés antiques (Robert 2006a et b). À Villliers-le-Sec, ils ont structuré l'habitat antique et médiéval autour d'un carrefour empierré dès le Ier siècle ap. J. C. qui reçut le cimetière. Au début de l'époque carolingienne, la voirie de Montmorency et le chemin de Villaine deviennent la «colonne vertébrale » de l'habitat organisé alors en village-rue (Wabont et al. 2006 : 439-443). La découverte d'un sceat anglosaxon frappé vers 725-730 et d'un denier frison de Dorestate (fin VIIe-début VIIIe s.) indique par ailleurs que ce site était peut-être en relation avec les ports du Nord.

À Marines, le « chemin de Traverse » entrant dans l'itinéraire Paris-Dieppe a été fouillé par C. Devals. Un niveau de circulation caractérisé par la présence d'ornières a livré du matériel du haut Moyen-Âge, médiéval et moderne. Ce chemin ne recoupe aucune des structures d'habitat et des sépultures datées du VI ${ }^{\text {e }}$ siècle à la fin du $\mathrm{IX}^{\mathrm{e}}$ siècle ap. J. C. et observe une parenté d'orientation avec eux. Il est donc au moins contemporain du village mérovingien et

3 L. Gonzalo-Valencia op. cit.

4 Gentili, F. Villiers-le-Sec (Val d’Oise), « La Place de la Ville », SDAVO, SRA, AFAN, Saint-Denis, Mars 2000 . 
carolingien des "Carreaux"5.

Fig. 3 : Les découvertes de chemins du haut Moyen-Âge sont nombreuses lors des opérations d'archéologies préventives et montrent un réseau structuré pour cette période (Gentili 2000 : 166)

II - 3 : Voies médiévales et structuration du paysage

À l'échelle des territoires, plusieurs cas de transmission des réseaux routiers antiques dans le paysage médiéval ont été observés.

Dans la vallée de l'Oise, en aval de Compiègne, où de nombreuses observations archéologiques ont été réalisées ces dernières années, les archéologues estiment que « durant le haut Moyen-Âge les chemins et voies antiques entretenues ont continué d'être utilisés » (Malrain et al. 1996 : 36).

À Sénart, la fixation des noyaux d'habitat au Moyen-Âge, s'est faite sur des pôles souvent déjà occupés à l'Antiquité voire à la Protohistoire, à proximité du réseau hydrographique et du réseau viaire. Plusieurs voies de grand parcours structurant le parcellaire dès l'Antiquité, sont apparues comme particulièrement attractives pour l'habitat médiéval qu'ils s'agissent de noyaux paroissiaux ou d'écarts. Quelques voies semblent être des créations médiévales générées par la polarisation autour de certains sites. Mais il s'agit d'axe de desserte locale et ils n'induisent pas de refonte des orientations parcellaires mises en place à l'Antiquité. Par exemple, l'orientation lisible sur le cadastre napoléonien autour du site " Les Fourneaux » à Vert-Saint-Denis était déjà présente dans les structures d'habitats antiques, mérovingiennes et carolingiennes. Elle s'insérait dans deux bandes parcellaires légèrement ondulées s’appuyant sur l'ancienne route de Melun à Brie, dans l'itinéraire Sens-Meaux. Une nouvelle voie dessert le site à partir Xe siècle. Large d'environ $7 \mathrm{~m}$ et bordée de fossés, elle a fait l'objet de plusieurs recharges constituées de cailloutis mêlé à du limon. Son orientation sud-est/nord-est diverge fortement par rapport à celle présente jusqu'alors. Malgré sa largeur, les archéologues l'ont interprété comme une desserte locale (Daveau et Goustard 2000). Effectivement, l'étude archéogéographique montre qu’il fait partie du réseau étoilé qui s'est développé autour du hameau de Pouilly-le-Fort, se superposant aux trames linéaires et quadrillé plus anciennes (Robert 1996). Ce hameau dépendant de Vert-Saint-Denis, s'est développé autour du château et de la chapelle de Pouilly sur le gué d'un affluent de la Seine. Sa première mention date de 1230 (Hubert 1930) mais la datation du chemin par la fouille des Fourneaux montre qu'il pourrait être plus ancien. Outre, qu'il s'agit d'un des rares cas de datation par la fouille d'un axe d'un réseau étoilé médiéval, cet exemple nous renseigne aussi sur la constitution d'une trame parcellaire radio-concentriques. En effet, on lit sur une photographie aérienne de l'IGN datant de 1963, une auréole de parcelles s’appuyant sur le réseau étoilé avec en son centre le hameau de Pouilly-le-Fort. Mais elle ne date - ... que des remembrements des années 1950 où les parcelles ont été réorientées sur les chemins locaux alors qu'au XIXe siècle, le parcellaire s'appuyait toujours sur la voie de grand parcours antique (Robert 1997 : 89).

À Sénart, le regroupement villageois au Moyen-Âge ne remet donc pas en question le réseau de circulation des voies de grand parcours hérité de l'Antiquité. Celui-ci continue à être utilisé et à influencer l'implantation de l'habitat mais avec d'autres critères : continuité avec une occupation ancienne, proximité au réseau hydrographique, implantation sur des limites fortes des réseaux préexistants, carrefour de voies.

Ailleurs, le croisement entre l'analyse archéogéographique et les données de terrain indique au contraire des bifurcations dans les réseaux parcellaires et la distribution de l'habitat. Elles

5C. Devals : Marines-Santeuil - Les Carreaux. Document final de synthèse de fouille préventive de mars à août 2001. Saint-Denis, Pantin, DRAC-SRA ILe-de-France, INRAP, CG-Val-d'Oise, 2003 : 83-85 
semblent répondre à des transformations dans les réseaux de circulation de grand parcours.

À Marines, par exemple, deux enclos emboîtés, datés de la Tène finale et du Ier siècle ap. J. C. présentaient une orientation semblable à celle de la chaussée romaine Orléans-Beauvais alors qu'ils n'entretenaient aucune relation avec le " chemin de Traverse », dans l'axe ParisDieppe, pourtant situé à une cinquantaine de mètre du site. En revanche, sur le village du haut Moyen-Âge "Les Carreaux », l’influence de la chaussée antique ne se fait plus sentir et les structures d'habitat et les sépultures datées du VI ${ }^{\mathrm{e}}$ siècle à la fin du $\mathrm{IX}^{\mathrm{e}}$ siècle ap. $\mathrm{J}$. C. présentent une parenté d'orientation avec le « chemin de Traverse » qui est structure le site. Par ailleurs, la chaussée romaine n'est plus construite en dur au delà de la période antique et ne semble plus attirer de points d'occupation. Ce changement dans les relations entretenues entre la voie antique et les établissements humains semble indiquer une prégnance plus importante de l'itinéraire Paris-Dieppe au détriment de celui d'Orléans-Beauvais à partir du haut Moyen-Âge. Le développement de cet itinéraire peut notamment s'expliquer par le développement du commerce du poisson à partir du haut Moyen-Âge. Des chemins de chassemarée traversant le Vexin en direction de Paris, sont attestés à Pontoise et Cergy ${ }^{6}$.

Ce type de phénomène était visible aussi sur l'opération Toyota-Onnaing où l'orientation nord-est/sud-ouest de la voie des Postes, axe arpentée, structurant pour les occupations du I siècle ap. J. C., n’apparaissait plus que de manière résiduelle sur le cadastre napoléonien. Les établissements et l'orientation du bâti pour les périodes médiévales et modernes se recentraient autour du faisceau est-ouest de l'itinéraire Valenciennes-Mons ${ }^{7}$.

D’après les observations archéologiques sur le terrain, le haut Moyen-Âge apparaît donc comme une période clé dans la transmission ou la bifurcation des réseaux. Dès 1982, M. Rouche signalait que «l'histoire mérovingienne donne la clef de l'évolution du système routier romain dans le Nord de la France » (Rouche 1982 : 85). La transformation des centres de décision politiques au nord de la Loire à partir de la période mérovingienne et carolingienne semble bien s'accompagner de réorientations dans le réseau routier de grand parcours. Le traditionnel axe nord-sud antique est concurrencé par un axe est-ouest reliant les nouvelles capitales et le débouché vers les ports de la Manche, ce qui rend fortement actif les itinéraires qui y mènent. À l'instar de ce qu'observe F. Bréchon dans le Vivarais, une sélection semble s’opérer sur les tracés antiques en fonction de leur orientation.

Le développement de nouveaux pôles peut venir court-circuiter aussi des tracés directs. À Marines, la déshérence de l'itinéraire Orléans-Beauvais, par le Vexin peut s'expliquer aussi par le développement de Paris qui capte l'itinéraire d'Orléans (cf. S. Robert infra). Ces réorganisations des flux sont lisibles aussi dans le sud de la France. À Lunel-Viel, par exemple, la modélisation des relations entre les voies et les sites archéologiques permet de lire des mutations dans l'orientation des flux importants en liaison avec l'émergence de nouveaux pôles économiques et politiques (Favory et al. 1994 : 208).

Pour certains chercheurs, le Moyen-Âge classique et final apparaît parfois comme une phase de renouveau routier liée aux performances techniques (Livet 2003 : 85) et au réengagement du pouvoir royal fort dans « un effort de construction et d'entretien des grandes routes » (Balard dir. 2003 : 234).

Pourtant, même pour les parcellaires de fondation développés autour des bastides de Gascogne qui constituent des cas de réorganisations parcellaires, C. Lavigne note qu'elles «s'adaptent, de manière très souple, à la topographie, au réseau hydrographique et aux chemins préexistants » (Lavigne 1997: 150). Des chemins de grand parcours, appelés

6 Robert, S. Étude de la structure paysagère ancienne de la commune de Cergy. Cergy, Mairie de Cergy,1999, 47 p.

7 Catted, I. coord., 1998. Rapport de fouilles archéologiques réalisées sur le site de Toyota. Lille : SRA, AFAN, Communauté de Communes de la Vallée de l’Escaut : 260-261. 
poudges ou pouches, implantés sur les replats de versants des affluents de la Garonne ont déterminé des lignes de forces dans le paysage servant d'armatures à des parcellaires de formation constitués dans la longue durée. Certaines de ces limites ont été reprises et réactivées dans les planifications médiévales (Lavigne 1997 : 155-157).

Enfin la période moderne, qui apparaît, après Rome, comme la deuxième véritable période de construction routière (Livet 2003), s'appuie en grande partie sur des itinéraires et des tracés hérités de l’Antiquité et du Moyen-Âge. À Sénart et dans le Val-d’oise, les routes royales ont toutes pour origine un itinéraire antique ou médiéval : soit elles s’appuient directement sur les tracés anciens, soit elles les redressent pour constituer un tracé plus rectiligne ou éviter certains obstacles.

\section{Conclusion}

Ces exemples montrent qu'il est nécessaire de bien prendre en compte les échelles de pratiques dans l'analyse des réseaux routiers. Les rythmes ne sont pas les mêmes à l'échelle des itinéraires régionaux et à celle des tracés locaux. La mobilité ou la pérennité qui s’observe à une échelle ne peut se généraliser à l'autre. De la même manière, la trame parcellaire a des temporalités qui lui sont propres et qui peuvent répondre ou non et avec un certain décalage aux transformations observées dans le réseau viaire.

Les observations réalisées sur le terrain sont nombreuses, surtout dans le contexte de l'archéologie préventive, mais demandent à être inventoriées et surtout à être comparées avec les données archéogéographiques pour pouvoir dépasser la fenêtre de la fouille et être hiérarchisées. On le voit les critères matériels seuls ne peuvent permettre de déterminer si l'on se trouve en présence ou non d'une voie de grand parcours. Sa matérialité peut changer en fonction des époques mais aussi des contextes. Plus soigné en contexte d'habitat, le même axe routier peut présenter une physionomie très différente dans la campagne.

\section{Bibliographie}

AGACHE, R. 1978. La Somme pré-romaine et romaine. Amiens, Société des Antiquaires de Picardie, 515 p.

BALARD, M. dir., LAURIOUX, B. LE JAN, R., LE MENÉ, M. 2003. Dictionnaire de la France médiévale. Paris, Hachette, 287 p.

BAUTIER, R. H. 1987. "La route française et son évolution au cours du Moyen Age », Académie royale de Belgique, Bulletin de la classe des lettres et sciences morales et politiques, 1987, t. 73 : 70-104.

BRÉCHON, F. 2000. Réseau routier et organisation de l'espace en Vivarais et sur ses marges au Moyen-Age. Thèse d'histoire. Lyon, Université de Lumière Lyon 2, 1368 p.

BRUAND, O. 2002. Voyageurs et marchandises aux temps carolingiens: les réseaux de communication entre Loire et Meuse aux VIIIe et IXe siècles. Bruxelles, De Boeck Université, 357 pages

CHAPOTAT, G. 1981. «La voie protohistorique sud de la croisée de Vienne. Essai de reconstitution de son tracé jusqu'à Marseille ». Revue Archéologique de l’Est, 1981, Tome XXXII, nº125-126 : 83-91

CHARTRAIN, A. 2007. «Parcellaires fossiles et faits funéraires dans l'espace et le temps sur le territoire rural de Montpellier » Medieval Europe Paris 2007, http://www.archeogeographie.org

CHEVALLIER, R. 1972. Les voies romaines. Paris, Armand Colin, 313 p.

CHEVALLIER, R. dir. 1983. « Les voies anciennes en Gaule et dans le monde romain occidental, Colloque tenu à Paris, ENS, 5-6 juin 1982 ». Caesarodunum nXVIII, Tours, Université de Tours, 487 p. 
CHOUQUER, G. et al. 1996. «La morphologie agraire et les paysages de la plaine des Tilles et de l'Ouche (Côte-d'Or) ». Chouquer, G. dir. 1996 : 32-49

CHOUQUER, G. dir. 1996. Les Formes du paysage, Tome I : Études sur les parcellaires : pré-actes du colloque d’Orléans (mars 1996). Paris, Éditions Errance, 222 p.

CHOUQUER, G. 1996. "Parcellaires et longue durée; Points de repères historiques et problèmes d'interprétation ", in: Chouquer G. dir. Les Formes du paysage, Tome 2 : Archéologie des parcellaires. Actes du colloque d'Orléans (mars 1996). Paris, Editions Errance, 1996 : 201-223

CHOUQUER, G. dir. 1997. Les Formes du paysage, Tome III : L'analyse des systèmes spatiaux. Paris, Éditions Errance, 198 p.

CHOUQUER, G. 2006. "L'émergence de la planimétrie agraire à l'Age du Fer », in : Chouquer G. dir. Etudes Rurales $n^{\circ}$ 175-176, Paris, Ecole des Hautes Etudes en Sciences Sociales, 2006 : 29-52

DAVEAU, I. ET GOUSTARD, V. « L'extraction et la réduction du minerai de fer à Vert-Saint- Denis (Seine-etMarne) durant le haut moyen âge : l'émergence d'une "industrie lourde" au sein d'un habitat à vocation agricole » in: Arts du feu et productions artisanales : Actes des XXe rencontres d'Antibes. Antibes : APDCA, 2000, pp. 1536

DAVEAU, I. et YVINEC, J. H. 2002. « L'occupation gallo-romaine du site de Fontenay-en-Parisis "La Lampe" (Val-d'Oise) : établissement agricole spécialisé ou lieu de culte ? » in : Revue Archéologique du Centre, Tome 41, Tours, $2002: 129-172$

DERVILLE, A. 1978. «La première révolution des transports continentaux, (c.1000 - c. 1300.) », in : Annales de Bretagne et des Pays de l'Ouest 85 (1978), Rennes : 181-205

DESBORDES, J. M. et BARRIÈRE, B. 1983. « Anciens itinéraires entre Limousin et Périgord », in : Chevallier R. (dir.) 1983 : 189-199

DEVROEY, J. P. 1984. « Un monastère dans l'économie d'échanges : les services de transport à l'abbaye SaintGermain-des-Prés au IXe siècle », in : Annales ESC 39, 1984 : 570-589

DUNIN-WASOWICZ, T. 1982. « Les routes médiévales en Europe centrale : bilan et perspectives », in : Flaran $1982: 283-288$

FAVIER, J. 1993. Dictionnaire de la France médiévale. Paris, Fayard, 982 p.

FAVORY, F., PARODI, A., POUPET, P. et RAYNAUD, C. 1994. « Lunel-Viel et son territoire », in : Favory F. et Fiches J. L. (dir.). Les campagnes de la France méditerranéenne dans l'Antiquité et le haut Moyen-Age, DAF $n^{\circ} 2$, Paris, Editions de la maison des sciences de l’Homme, 1994 : 163- 244

FLARAN, 1982. "L'homme et la route en Europe occidentale au Moyen Age et aux Temps Modernes », in : Cahier de Flaran, $n^{\circ}$ 2, Auch, 1982, 303 p.

GRENIER, A. 1985. Manuel d'archéologie gallo-romaine. Tome 2, L'archéologie du sol. Navigation-occupation

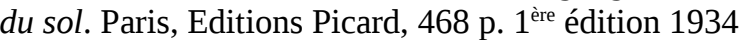

GUILLAUMET, J. P. 1996. L'artisanat chez les gaulois. Paris, Errance, 127 p.

JULLIAN, C. 1920. Histoire de la Gaule, tome V, Paris, Hachette.

JUNG, C. 1999. Morphogénèse, fonctions et évolution de la centuriation B d'Orange et essai de restitution diachronique des paléopaysages du Tricastin (Drôme-Vaucluse). Thèse pour obtenir le grade de Docteur de l'Université de Tours.Tours, Université de Tours, 366 p.

LAMBOT, B. 1996. «Les Rèmes à la veille de la romanisation. Le porcien au Ier siècle avant J. C. ", in : Bayard D. et Collard J. L. (dir.), Revue Archéologique de Picardie, $n^{\circ}$ spécial, 11/1996, Amiens : 13-38 
LAFFONT, P. Y et BRÉCHON, F. 2008. « Châteaux, incastellamento et réseau viaire au Moyen-Age en France méridionale », in : Conpatangelo-Soussignan R., Bertrand J. R., Chapman J. et Laffont P. Y. (dir.). Marqueurs des paysages et systèmes socio-économiques. Actes du colloque COST du Mans (7-9 décembre 2006), Rennes, Presses Universitaires, 2008 : 149-163

LAVIGNE, C. 1997. « Parcellaires de fondation et parcellaires de formation à l'époque médiévale en Gascogne. Clefs de lecture et problèmes d’interprétation », in : Chouquer G. (dir.) 1997 : 149-159

LEBECQ, S. 1989. « La Neustrie et la mer », in : H. Atsma (publ.), La Neustrie, Les pays au nord de la Loire de 650 à 850. Jan Thorbecke Verlag Sigmaringen, 1989 : 406-440

LEGROS, R. 1983. « Les chemins des Rèmes », in : Chevallier R. (dir.) 1983 : 269-289

LIVET, G. 2003. Histoire des routes et des transports en Europe. Des chemins de Saint-Jacques à l'âge d'or des diligences. Strasbourg, Presses universitaires, 608 p.

MALRAIN, F., MATTERNE, V., MÉNIEL, P. 2002. Les paysans gaulois. Paris, Errance, 2002, 236 p.

MENNESSIER-JOUANNET, C. et BUCHSENSCHUTZ, O. 1996. "Des “champs celtiques” aux systèmes de parcellaire protohistoriques », in : Chouquer G. (dir.) 1996 : 174-180

ROBERT, S. 1996. « Le Parcellaire du plateau de Sénart (Seine-et-Marne) », in : Chouquer G. (dir.) 1996 : 11 27.

ROBERT, S. 1997. « Le relevé parcellaire sur photographies aériennes et cartes anciennes », in : Chouquer G. (dir.) $1997: 88-95$

ROBERT, S. 2006a. «La résilience des réseaux routiers : l'exemple du Val-d'Oise », in : Bulletin AGER $n^{\circ} 15$, année 2005, Besançon, Presses Universitaires de Franche-Comté, 2006 : 8-14

ROBERT, S. 2006b. "Les itinéraires anciens traversant le département du Val-d’Oise », in : Bulletin archéologique du Vexin Français et du Val-d’Oise, n³8 : 7-23

ROUCHE, M. 1982. "L’héritage de la voirie antique dans la Gaule du haut Moyen-Age (Ve-XIe siècle) », in : Flaran 1982 : 12-32

VOYAGES ET VOYAGEURS AU MOYEN AGE, 1996. Société des historiens médiévistes de l'enseignement supérieur public. Voyages et Voyageurs au Moyen Age. XXVIe Congrès de la SHMES (Limoges-Aubazine, mai 1995), Paris, Publications de la Sorbonne, 314 p.

WABONT, M., ABERT, F., VERMEERSCH, D. 2006. Le Val-d’Oise - 95. In : Provost M. (dir.), Carte Archéologique de la Gaule. Le département du Val-d'Oise. Paris : Editions Les Belles Lettres, 495 p.

WATTEAUX, M. 2004. «Le plan radio-quadrillé des terroirs non planifiés », in : Chouquer G. (prés.). Etudes Rurales $n^{\circ} 167-168$. Paris, EHESS, 2004 : 187-214.

VIDAL, L., POMARÈDES, H. et SAUVAGE, L. 1996. «Traces matérielles de la mise en valeur et de l'aménagement des campagnes aux portes de Nîmes : les parcellaires antiques de la plaine du Vistre ", in : Chouquer G. (dir.) : Les Formes du paysage, Tome II : Études sur les parcellaires : actes du colloque d'Orléans (mars 1996). Paris, Éditions Errance, 1996 : 57-66 